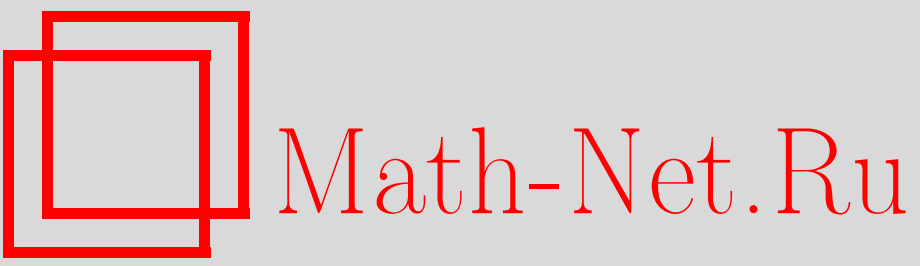

А. А. Арсеньев, Резонансы и туннелирование при рассеянии на квантовом бильярде в приближении сильной связи, ТМФ, 2004, том 141, номер 1, 100-112

DOI: https://doi.org/10.4213/tmf110

Использование Общероссийского математического портала Math-Net.Ru подразумевает, что вы прочитали и согласны с пользовательским соглашением

http://www . mathnet.ru/rus/agreement

Параметры загрузки:

IP: 52.6 .47 .48

26 апреля 2023 г., 17:24:02 
ТЕОРЕТИЧЕСКАЯ

И МАТЕМАТИЧЕСКАЯ

ФИЗИКА

Том 141, № 1

октябрь, 2004

(C) 2004 г.

А. А. Арсеньев*

\section{РЕЗОНАНСЫ И ТУННЕЛИРОВАНИЕ ПРИ РАССЕЯНИИ НА КВАНТОВОМ БИЛЬЯРДЕ В ПРИБЛИЖЕНИИ СИЛЬНОЙ СВЯЗИ}

\footnotetext{
В приближении сильной связи рассмотрена задача рассеяния на квантовом бильярде и исследовано поведение матрицы рассеяния вблизи резонанса.
}

Ключевые слова: модель сильной связи, квантовый волновод, резонанс.

\section{1. ВВЕДЕНИЕ}

Модель сильной связи является вариантом теории, рассматривающей в качествепространства состояний гильбертово пространство функций на целочисленной решетке. Данная модель возникает как одна из простейших в физике твердого тела: считается, что каждый атом взаимодействует только с ближайшим соседом и не имеет внутренней структуры. Кроме того, эта модель применяется при разностной аппроксимации уравнения Шредингера. Иногда на основе модели сильной связи удается получить описание реальных опытов, но особенно она популярна при проведении численных экспериментов и при проверке теоретических предположений в физике твердого тела.

Еще в первых работах по теории туннельного диода [1] было отмечено, что симметрия системы сушественно влияет на физику процесса туннелирования. Позже факт влияния симметрии на туннелирование стал общепризнанным. В работе [2] на связь между туннелированием и симметрией обрашено внимание при обсуждении результатов численного эксперимента по рассеянию в модели сильной связи. В нашей работе мы рассматриваем задачу рассеяния в модели сильной связи и исследуем влияние симметрии системы на рассеяние путем анализа резонансов и туннелирования в системах со связанными состояниями (системах $2 \times 2$ ) [3], [4].

Рассмотрим систему, состоящую из цепочки $2 n+1$ атомов (бильярд), которая присоединена к бесконечному проводнику. Докажем следуюшие утверждения. Пусть коншы $L$ и $R$ бильярда соединены последовательно с идущим из $-\infty$ в $L$ и из $+\infty$ в $R$ проводниками, и система симметрична относительно точки 0 . Тогда в случае слабой связи биль-

* Московский государственный университет, Москва, Россия. E-mail: arsenev@afrodita.phys.msu.su 
ярда с проводником есть резонансное туннелирование: на резонансных частотах бильярда волна будет проходить из $-\infty$ в $+\infty$ почти без отражения, а на других частотах она будет почти полностью отражаться. Если же бильярд в точках $L$ и $R$ присоединен параллельно к идущему из $-\infty$ в $+\infty$ проводнику, то в случае слабой связи бильярда с проводником на резонансных частотах бильярда волна будет почти полностью отражаться, а на других частотах она будет проходить без отражения ${ }^{1)}$.

В численных экспериментах описанная выше картина рассеяния хорошо известна и обнаруживается достаточно просто (см., например, [6]), и вопрос состоит в том, чтобы дать фактам численного эксперимента теоретическое толкование. Такое толкование мы даем на основе предложенной в работе [3] модели. Эта модель позволяет объяснить качественно разное поведение матрицы рассеяния вблизи резонансов бильярда в случае параллельного и последовательного соединения и объяснить влияние симметрии на картину рассеяния. Основной результат работы изложен ниже в теоремах 3 и 4.

\section{2. ЗАДАЧА РАССЕЯНИЯ}

Формула Ландауэра связывает баллистическую проводимость с квадратом нормы Гильберта-Шмидта минора матрицы рассеяния, составленного из коэффициентов прохождения. Поэтому изучение баллистической проводимости сводится к изучению матрицы рассеяния, составленной из коэффициентов прохождения и отражения. В математической теории рассеяния под матрицей рассеяния понимается матрища оператора рассеяния в базисе из собственных функций непрерывного спектра невозмущенного оператора. Обычно связь между этими двумя понятиями матрицы рассеяния устанавливается так: матричный элемент вычисляется интегрированием по частям и выражается через асимптотику “искаженных плоских волн”. В модели сильной связи этот способ не работает, так как матричный элемент выражается суммой, а не интегралом. Мы покажем, что в модели сильной связи интегрирование по частям при вычислении матричного элемента матрицы рассеяния можно заменить суммированием по Абелю. Соответствующий прием достаточно пояснить на одномерном примере, что и будет сделано в этом разделе.

Пусть $l$ - линейное пространство бесконечных в обе стороны последовательностей комплексных чисел:

$$
f \in l, \quad f: \mathbb{Z} \ni j \rightarrow f(j) \in \mathbb{C}^{1} .
$$

В $l$ выделим гильбертовы пространства $l^{2}, l_{a}$, в которых квадрат нормы задается равенствами

$$
\left\|f\left|l^{2}\left\|^{2}=\sum_{j}|f(j)|^{2}, \quad\right\| f\right| l_{a}^{2}\right\|^{2}=\sum_{j}|f(j)|^{2} e^{-a|j|} .
$$

Будем считать, что скалярное произведение $\langle\cdot, \cdot\rangle$ в $l^{2}$ линейно по второму аргументу и расширено до двойственности между $l_{a}^{2}$ и $l_{-a}^{2}$. Пространства $l_{a}^{2}$ и $l_{-a}^{2}$ мы будем рассматривать как обычно используемое в теории рассеяния оснащение исходного гильбертова

\footnotetext{
${ }^{1)} \mathrm{K}$ аналогичным выводам пришли авторы работы [5].
} 
пространства. Пусть $\left\{A_{j}\right\},\left\{B_{j}\right\}$ - ограниченные последовательности действительных чисел. Очевидна

Лемма 1. Onератор

$$
L: L f(j)=A_{j+1} f(j+1)+A_{j} f(j-1)-\left(A_{j+1}+A_{j}-B_{j}\right) f(j)
$$

самосопряжен в $l^{2}$. Если

$$
A_{-j}=A_{j+1}, \quad B_{-j}=B_{j},
$$

то оператор $L$ коммутирует с оператором отражения

$$
I: I f(j)=f(-j)
$$

Положим

$$
L_{0} f(j)=-f(j+1)-f(j-1)+2 f(j) .
$$

Оператор $L_{0}$ мы будем рассматривать как невозмущенный гамильтониан. Такой выбор невозмушенного гамильтониана отличается от обычно используемого в физике твердого тела знаком и сдвигом на константу и обусловлен желанием сделать выкладки и окончательные формулы максимально похожими на те, которые встречаются при описании задачи рассеяния на прямой для оператора $-D_{x x}^{2}$. Заметим, что с точностью до множителя оператор (5) есть аппроксимация оператора $-D_{x x}^{2}$ центральной разностью на равномерной сетке.

Преобразование

$$
F: l^{2} \rightarrow L^{2}([0,1]), \quad F(f)(x)=\sum_{j} f(j) e^{2 \pi i j x}, \quad x \in[0,1],
$$

унитарно, и обратное преобразование дается формулой

$$
F^{-1}: f(j)=\int_{0}^{1} F(f)(x) e^{-2 \pi i j x} d x .
$$

В $L^{2}([0,1])$ образ оператора $L_{0}$ есть оператор умножения на функцию

$$
\omega(x)=2(1-\cos (2 \pi x)) .
$$

Отсюда следует, что спектр $\sigma\left(L_{0}\right)$ оператора $L_{0}$ есть отрезок $[0,4]$ и абсолютно непрерывен. Из (6)-(8) следует, что резольвента $R\left(\lambda, L_{0}\right)=\left(\lambda-L_{0}\right)^{-1}$ оператора $L_{0}$ дается формулой

$$
R\left(\lambda, L_{0}\right) f(j)=\sum_{m}\left[\int_{0}^{1}(\lambda-\omega(x))^{-1} e^{2 \pi i(m-j) x} d x\right] f(m) .
$$


Вычисляя этот интеграл, получаем

$$
\left(R\left(\lambda+i 0, L_{0}\right) f\right)(j)=(2 \sin (k(\lambda)))^{-1} \sum_{m} e^{i k(\lambda)|m-j|} f(m), \quad 0<\lambda<4,
$$

где

$$
k(\lambda)=\arccos \left(1-\frac{\lambda}{2}\right), \quad 0<k(\lambda)<\pi, \quad 0<\lambda<4 .
$$

Используя известную формулу для разложения единицы

$$
d E\left(\lambda, L_{0}\right) f=(2 \pi i)^{-1}\left(R\left(\lambda-i 0, L_{0}\right)-R\left(\lambda+i 0, L_{0}\right)\right) f d \lambda
$$

мы получаем

$$
d E\left(\lambda, L_{0}\right) f=(2 \pi \sin (k(\lambda)))^{-1}\left[\sum_{m} \cos ((m-j) k(\lambda)) f(m)\right] d \lambda .
$$

Эта формула дает спектральное представление оператора $L_{0}$.

Преобразуем его в удобную для нас форму, чтобы иметь возможность применить результаты работы [3]. Пусть $\Omega=\{+,-\}-$ двухточечное множество. Будем рассматривать его как пространство с мерой $d \omega$, приписав каждой точке меру +1 . Пусть $h-$ гильбертово пространство функций на отрезке $[0,4]$ со значениями в $L^{2}(\Omega, d \omega)$,

$$
\|\phi \mid h\|^{2}=\int_{0}^{4}\left[\int_{\Omega}|\phi(\lambda, \omega)|^{2} d \omega\right] d \lambda .
$$

Представив в (12) косинус через экспоненты и используя известные свойства разложения единицы

$$
f=\int_{\sigma\left(L_{0}\right)} d E\left(\lambda, L_{0}\right) f, \quad\|f\|^{2}=\int_{\sigma\left(L_{0}\right)}\left\langle f, d E\left(\lambda, L_{0}\right) f\right\rangle,
$$

мы из (12) получим следуюшее утверждение.

ЛЕмма 2. Преобразование

$$
U: l^{2} \rightarrow h, \quad(U f)(\lambda, \pm)=(4 \pi \sin (k(\lambda)))^{-1 / 2} \sum_{m} e^{\mp i m k(\lambda)} f(m)
$$

унитарно, и обратное преобразование дается формулой

$$
\begin{aligned}
U^{-1}: f(j)= & \int_{0}^{4}\left(U(f)(\lambda,+) e^{i k(\lambda) j}+U(f)(\lambda,-) e^{-i k(\lambda) j}\right) \times \\
& \times(4 \pi \sin (k(\lambda)))^{-1 / 2} d \lambda .
\end{aligned}
$$

В обеих частях равенства (14) берутся одновременно либо верхние знаки, либо нижние, в формуле (15) $f$ - элемент пространства $l^{2}, U(f)(\lambda, \pm)$ - его образ в $h$.

Пусть оператор $V: l \rightarrow l$ задается своими матричными элементами:

$$
V f(m)=\sum_{p} V(m, p) f(p) .
$$


ЛЕмма 3. Если

$$
V(m, p)=\overline{V(p, m)}, \quad|V(m, p)|<\mathrm{const} \cdot e^{-b(|m|+|p|)}, \quad b>0,
$$

то $V$ - ядерный оператор в $l^{2}$. При $b>a>0$ оператор $V$ вполне непрерывен как оператор из $l_{a}^{2}$ в $l_{-a}^{2}$.

ДокАЗАТЕльство. В пространстве $L^{2}([0,1])$ оператор $V$ есть интегральный оператор с эрмитовым бесконечно дифференцируемым периодическим ядром, и поэтому (см. [7], с. 154-155) он ядерный. Второе утверждение тривиально.

Положим

$$
L=L_{0}+V \text {. }
$$

Определим функции $e(\lambda): \Omega \times \mathbb{Z} \rightarrow \mathbb{C}, e(\lambda, \pm, j)=e^{ \pm i k(\lambda) j}$. Функцию $u(\lambda): \Omega \times \mathbb{Z} \rightarrow \mathbb{C}$ мы назовем решением задачи рассеяния, если она удовлетворяет уравнению

$$
L u(\lambda)=\lambda u(\lambda)
$$

и представима в виде

$$
u(\lambda)=e(\lambda)+w(\lambda)
$$

где функция $w$ удовлетворяет условиям излучения:

$$
\begin{array}{ll}
w(\lambda,+, j)=(t(+, \lambda)-1) e(\lambda,+, j)+o(\ldots), & j \rightarrow+\infty, \\
w(\lambda,+, j)=r(+, \lambda) e(\lambda,-, j)+o(\ldots), & j \rightarrow-\infty, \\
w(\lambda,-, j)=r(+, \lambda) e(\lambda,+, j)+o(\ldots), & j \rightarrow+\infty, \\
w(\lambda,-, j)=(t(-, \lambda)-1) e(\lambda,-, j)+o(\ldots), & j \rightarrow-\infty,
\end{array}
$$

и $о(. .$.$) означает, что$

$$
\exists \delta>0: o(\ldots)=O\left(e^{-\delta|j|}\right), \quad|j| \rightarrow \infty .
$$

Коэффициенты $t( \pm, \lambda)$ и $r( \pm, \lambda)$ в формулах $(20)$ мы будем называть коэффициентами прохождения и отражсения соответственно.

ЛЕмма 4. Если функиия $w$ удовлетворяет условиям излучения, то для любого $j$

$$
\epsilon R\left(\lambda+i \epsilon, L_{0}\right) w(\lambda, \pm, j) \rightarrow 0, \quad \epsilon \rightarrow 0
$$


ДОКАЗАТЕЛЬСтво. Разберем для простоты только случай положительного знака в левой части (21). Подставим $(20)$ как $f(m)$ в $(9)$ и разобьем сумму в правой части $(9)$ на три суммы: по индексам $|m| \leqslant|j|, m<-|j|, m>|j|$. Для первой суммы при фиксированном $j$ утверждение леммы очевидно. В третьей сумме оценка содержащих члены $o(\ldots)$ слагаемых также очевидна, а оставшаяся сумма есть сумма геометрической прогрессии со знаменателем

$$
q(\epsilon)=e^{i \operatorname{Re} k(\lambda+i \epsilon)-\operatorname{Im} k(\lambda+i \epsilon)} .
$$

Так как $\varlimsup_{\epsilon \rightarrow 0}\left(|1-q(\epsilon)|^{-1}\right)<\infty$, то для третьей суммы утверждение леммы верно. Оценка второй суммы аналогична.

Положим

$$
\Gamma(\lambda)=R\left(\lambda, L_{0}\right) V, \quad \operatorname{Im} \lambda>0, \quad 0<\operatorname{Re} \lambda<4 .
$$

Лемма 5. Если оператор $V$ удовлетворяет оценке (16), то при $0<a<b$ операторнозначная функция

$$
\lambda \rightarrow \Gamma(\lambda) \in L\left(l_{a}^{2} \rightarrow l_{a}^{2}\right)
$$

аналитична в некоторой комплексной окрестности интервала $(0,4)$, и ее значения суть вполне непрерывные операторы.

Здесь и далее символом $L\left(\mathfrak{B}_{1} \rightarrow \mathfrak{B}_{2}\right)$ мы обозначаем банахово пространство всех линейных непрерывных операторов из банахова пространства $\mathfrak{B}_{1}$ в банахово пространство $\mathfrak{B}_{2}$.

Для доказательства леммы достаточно заметить, что предел по норме конечномерных операторов есть вполне непрерывный оператор.

Лемма 6. Функиия и $(\lambda)$ есть решение задачи рассеяния в том и только том случае, когда функиия $w(\lambda)$ в (19) есть решение уравнения

$$
w(\lambda)=\Gamma(\lambda)(e(\lambda)+w(\lambda)) .
$$

ДокАЗАТЕльСтво. Пусть функция $u$ в (19) есть решение задачи рассеяния. Тогда из уравнения (18) следует

$$
\left(\lambda-L_{0}\right) w(\lambda)=V(e(\lambda)+w(\lambda))
$$

и

$$
R(\lambda+i \epsilon) V(e(\lambda)+w(\lambda))=w(\lambda)-i \epsilon R\left(\lambda+i \epsilon, L_{0}\right) w(\lambda) .
$$

Переходя в последнем равенстве к пределу при $\epsilon \rightarrow+0$ и учитывая лемму 4 , мы получаем (24). Если функция $w$ есть решение уравнения (24), то она удовлетворяет условиям излучения в силу формулы (10). Так как в комплексной окрестности интервала $(0,4)$ выполнено равенство

$$
\left(\lambda-L_{0}\right) \Gamma(\lambda)=V,
$$

то из уравнения (24) следует уравнение (18). Лемма доказана. 
ЛЕмма 7. При $\lambda \in(0,4)$ решение уравнения

$$
w(\lambda)=\Gamma(\lambda) w(\lambda), \quad w(\lambda) \in l_{a}^{2},
$$

принадлежит $l^{2}$.

ДОКАЗАТЕЛЬСТво. Из (26) следует, что функция $w(\lambda)$ удовлетворяет уравнению (18), поэтому

$$
\begin{aligned}
0= & \sum_{|j| \leqslant m}(\overline{w(\lambda, j)} L w(\lambda, j)-w(\lambda, j) L \overline{w(\lambda, j)})= \\
= & \overline{w(\lambda, m+1)} w(\lambda, m)-\overline{w(\lambda, m)} w(\lambda, m-1)+ \\
& +\overline{w(\lambda,-m-1)} w(\lambda,-m)-\overline{w(\lambda,-m)} w(\lambda,-m-1)+O\left(e^{-\delta|m|}\right) .
\end{aligned}
$$

Если

$$
w(\lambda, j)=C_{ \pm} e^{i k(\lambda)|j|}+O\left(e^{-\delta|j|}\right), \quad j \rightarrow \pm \infty,
$$

то из (27) следует, что

$$
\left(e^{-i k(\lambda)}-e^{i k(\lambda)}\right)\left(\left|C_{+}\right|^{2}+\left|C_{-}\right|^{2}\right)+O\left(e^{-\delta|m|}\right)=0,
$$

поэтому в (28) $C_{ \pm}=0$, что и доказывает лемму.

Из лемм $5-7$ следует

Теорема 1. При $\lambda=\lambda_{0} \in(0,4)$ либо уравнение (18) имеет нетривиальное решение из $l^{2}$, либо решение задачи рассеяния существует, единственно и в достаточно малой окрестности точки $\lambda_{0}$ является аналитической функиией от $\lambda$ со значениями в $l_{a}^{2}$.

Пусть $\sigma_{d}(L)$ - множество тех значений $\lambda$, для которых уравнение (18) имеет нетривиальное решение в $l^{2}$.

Лемма 8. При $\lambda \notin \sigma_{d}(L)$ решение уравнения (24) дается формулой

$$
w(\lambda)=R(\lambda, L) V e(\lambda) .
$$

ДоКАЗАТЕЛЬСтво. Обозначим правую часть $(29)$ через $\widetilde{w(\lambda)}$ и докажем, что она удовлетворяет уравнению (24). Имеем

$$
\begin{aligned}
\Gamma(\lambda)(e(\lambda)+\widetilde{w(\lambda)}) & =R\left(\lambda, L_{0}\right) V e(\lambda)+R\left(\lambda, L_{0}\right) V R(\lambda, L) V e(\lambda)= \\
& =\left[R\left(\lambda, L_{0}\right)+R\left(\lambda, L_{0}\right) V R(\lambda, L)\right] V e(\lambda)= \\
& =R(\lambda, L) V e(\lambda)=\widetilde{w(\lambda)} .
\end{aligned}
$$

Так как решение уравнения (24) единственно, то лемма доказана.

Определим оператор

$$
Z(\epsilon): l \rightarrow l, \quad Z(\epsilon) f(j)=e^{-\epsilon|j|} f(j) .
$$

Прямым вычислением доказывается 
Лемма 9. Справедливо равенство

$$
\left(\lambda-L_{0}\right) Z(\epsilon) e(\lambda, \pm, j)=\mp 2 i\left(\sin (k(\lambda)) \epsilon \operatorname{sign}(j) e(\lambda, \pm, j) e^{-\epsilon|j|}+O\left(\epsilon^{2} e^{-\epsilon|j|}\right) .\right.
$$

Определим оператор

$$
T(\lambda): l_{a}^{2} \rightarrow l_{a}^{2}, \quad T(\lambda)=V+V R(\lambda, L) V, \quad \operatorname{Im} \lambda>0
$$

В окрестности каждой точки $\lambda \in(0,4) \backslash \sigma_{d}(L)$ оператор $T(\lambda)$ есть аналитическая функция от $\lambda$ со значениями в $L\left(l_{a}^{2} \rightarrow l_{a}^{2}\right)$.

Лемма 10. При $\lambda \notin \sigma_{d}(L)$ справедливо равенство

$$
T(\lambda) e(\lambda)=\left(\lambda-L_{0}\right) w(\lambda)
$$

ДокАЗАТЕЛЬСтво. С учетом равенств (29) и (18) имеем

$$
\begin{aligned}
T(\lambda) e(\lambda) & =V e(\lambda)+V[R(\lambda, L) V e(\lambda)]= \\
& =V e(\lambda)+V w(\lambda)=\left(L-L_{0}\right)(e(\lambda)+w(\lambda))=\left(\lambda-L_{0}\right) w(\lambda) .
\end{aligned}
$$

Определим волновые операторы и оператор рассеяния:

$$
\begin{aligned}
W_{ \pm}\left(L, L_{0}\right) & =\lim _{t \rightarrow \pm \infty} e^{i t L} e^{-i t L_{0}} \\
S\left(L, L_{0}\right) & =W_{+}^{*}\left(L, L_{0}\right) W_{-}\left(L, L_{0}\right) .
\end{aligned}
$$

Сушествование и полнота волновых операторов следуют из ядерности оператора $V$. Как известно [8], оператор рассеяния $S\left(L, L_{0}\right)$ коммутирует с оператором $L_{0}$, и поэтому в пространстве $h$ оператор рассеяния $S\left(L, L_{0}\right) \in L\left(L^{2}(\Omega) \rightarrow L^{2}(\Omega)\right)$ задается матрицей

$$
\left(U S\left(L, L_{0}\right) f\right)(\lambda)=S\left(L, L_{0}, \lambda\right)(U f)(\lambda)
$$

Теорема 2. Матрица $S\left(L, L_{0}, \lambda\right)$ в (33) выражсается через коэффичиенть прохождения и отражения следующим образом:

$$
S\left(L, L_{0}, \lambda\right)=\left(\begin{array}{cc}
t(+, \lambda) & r(-, \lambda) \\
r(+, \lambda) & t(-, \lambda)
\end{array}\right)
$$


ДокАЗАТЕЛЬСТво. Как известно [8], глава 2, раздел 8, формула (9), вычисление матрицы рассеяния сводится к вычислению матричных элементов оператора $T(\lambda)$ между функциями $e(\lambda, \pm)$. Найдем матричный элемент

$$
\begin{aligned}
& \langle e(\lambda,+), T(\lambda) e(\lambda,+)\rangle=\left\langle e(\lambda,+),\left(\lambda-L_{0}\right) w(\lambda,+)\right\rangle= \\
& \quad=\lim _{\epsilon \rightarrow+0}\left\langle Z(\epsilon) e(\lambda,+),\left(\lambda-L_{0}\right) w(\lambda,+)\right\rangle= \\
& \quad=\lim _{\epsilon \rightarrow+0}\left\langle\left(\lambda-L_{0}\right) Z(\epsilon) e(\lambda,+), w(\lambda,+)\right\rangle= \\
& \quad=-2 i \sin (k(\lambda))(t(+, \lambda)-1) .
\end{aligned}
$$

Заметим, что здесь во втором скалярном произведении нельзя перебросить оператор $\left(\lambda-L_{0}\right)$ на $e(\lambda,+)$, и поэтому мы ввели оператор $Z(\epsilon)$. Правая часть в $(35)$ получается на основе леммы 9 с последующим вычислением предела суммы геометрической прогрессии. Остальные матричные элементы вычисляются аналогично. Используя лемму 2, мы получаем утверждение теоремы.

Предыдущие результаты легко обобшаются на случай, когда элементы пространства $l$ суть вектор-функции со значениями в $\mathbb{C}^{n}$. В этом случае величины $A_{j}, B_{j}$ в $(2)$ есть самосопряженные операторы в $\mathbb{C}^{n}$, и $\omega(x)$ в $(8)$ - также оператор. Если для любого $x \in(0,1)$ все собственные значения оператора $\omega(x)$ простые, то дальнейшие обобщения очевидны и сводятся в основном к переосмыслению обозначений. Если оператор $\omega(x)$ имеет кратные собственные значения, то общий план рассуждений сохраняется, но необходим подробный анализ возможных случаев ветвления собственных значений.

С практической точки зрения вычисление матрицы рассеяния сводится (см. [9]) к следующей процедуре. В области $j \gg 1$ строят базис из “уходящих" волн, используя уравнение (18) как рекуррентное соотношение, этот базис перебрасывают в область $j \ll-1$ и полученные векторы разлагают по базису из "уходящих" и "приходящих" волн. Коэффициенты разложения и дают матрицу рассеяния. Трудностей здесь не возникает, если только спектральный параметр $\lambda$ не расположен близко к $\sigma_{d}$. Однако именно этот случай и интересен в приложениях. Если спектральный параметр $\lambda$ расположен близко к $\sigma_{d}$, то детерминант получающейся при использовании уравнения (18) системы линейных уравнений обрашается в нуль (это и есть наиболее надежный признак резонанса), и, как правило, программа решения системы линейных уравнений начинает вычислять “регуляризованное” решение, которое может не иметь ничего общего с искомым.

Две последуюшие леммы получаются прямым вычислением и приводятся без доказательств.

Лемма 11. Пусть оператор L задается формулой (2) с

$$
A_{j}=\left\{\begin{array}{l}
0, \quad-n \leqslant j \leqslant n+1 \\
-1, \quad j<-n, \quad j>n+1
\end{array}\right.
$$


Тогда решение задачи рассеяния дается формулой

$$
\begin{aligned}
& u(\lambda,+, j)= \begin{cases}e^{i k j}-e^{-2 i k n-i k j}, & j \leqslant-n-1, \\
0, & j \geqslant-n .\end{cases} \\
& u(\lambda,-, j)= \begin{cases}e^{-i k j}-e^{2 i k n+i k j}, & j \geqslant n+1, \\
0, & j \leqslant n .\end{cases}
\end{aligned}
$$

ЛЕмма 12. Пусть оператор L задается формулой (2) c

$$
A_{j}= \begin{cases}-1, & -n<j<n+1 \\ 0, & j \leqslant-n, \quad j \geqslant n+1 .\end{cases}
$$

Тогда спектр оператора $L$ дискретен и состоит из чисел

$$
\lambda_{m}=4 \sin ^{2}\left(\frac{\pi m}{4 n+2}\right), \quad 0 \leqslant m \leqslant 2 n,
$$

а собственнье функции суть

$$
\psi_{m}(j)=c_{m}\left(e^{i k_{m} j}+(-1)^{m} e^{-i k_{m} j}\right), \quad k_{m}=\frac{\pi m}{2 n+1}, \quad-n \leqslant j \leqslant n .
$$

Нормировочные константы $c_{m}$ нам не понадобятся, и мы их вычислять не будем. Заметим, что на собственные функции мы не налагаем никаких граничных условий, а требуем выполнения уравнения (18) для всех $j \in \mathbb{Z}$.

\section{3. ОСНОВНЫЕ РЕЗУЛЬТАТЫ}

В качестве фазового пространства $\mathcal{H}$ нашей системы мы возьмем пространство $l^{2}$ вектор-функций на $\mathbb{Z}$ со значениями в $\mathbb{C}^{2}$. Элементы $f(j) \in \mathbb{C}^{2}$ будем рассматривать как вектор-столбцы. Норму в $\mathcal{H}$ введем по формуле (1). Пространство $\mathcal{H}$ мы представим в виде прямой суммы:

$$
\mathcal{H}=\mathcal{H}_{1} \oplus \mathcal{H}_{2},
$$

где $\mathcal{H}_{1}$ - те функции из $l^{2}$, значения которых во вторых строках вектор-функций равны нулю, а $\mathcal{H}_{2}-$ те функции из $l^{2}$, значения которых в первых строках равны нулю. Гамильтониан $H_{0}$ невозмушенной системы (проводника) мы зададим формулой

$$
\left(H_{0} f\right)(j)=\left(\begin{array}{cc}
0 & 0 \\
0 & -1
\end{array}\right)(f(j+1)+f(j-1)-2 f(j)) .
$$

Систему проводник+бильярд мы будем описывать гамильтонианом

$$
H_{q}=H_{0}+V_{q}, \quad V_{q} \stackrel{\text { def }}{=} V_{\mathrm{box}}+\epsilon_{q} V_{\mathrm{int}},
$$

где

$$
\left(V_{\mathrm{box}} f\right)(j)=A_{j+1} f(j+1)+A_{j} f(j-1)-\left(A_{j+1}+A_{j}\right) f(j), \quad \epsilon_{q} \rightarrow 0, \quad q \rightarrow \infty .
$$


Будем считать, что оператор взаимодействия $V_{\text {int }}$ задается формулой

$$
\left(V_{\mathrm{int}} f\right)(j)=\left(\begin{array}{cc}
0 & \delta_{j}^{(-n)}+\delta_{j}^{(n)} \\
\delta_{j}^{(-n)}+\delta_{j}^{(n)} & 0
\end{array}\right) f(j),
$$

где $\delta_{j}^{(n)}$ - символ Кронекера.

В случае параллельного соединения бильярда с проводником оператор бильярда $V_{\text {box задается формулой }(39) \text { c }}$

$$
A_{j}=\left(\begin{array}{cc}
-g_{j} & 0 \\
0 & 0
\end{array}\right), \quad g_{j}=\left\{\begin{array}{l}
1,-n+1 \leqslant j \leqslant n, \\
0, j<-n+1, j>n .
\end{array}\right.
$$

В случае последовательного соединения бильярда с проводником оператор бильярда $V_{\text {box задается формулой }(39) \text { c }}$

$$
A_{j}=\left(\begin{array}{cc}
-g_{j} & 0 \\
0 & g_{j}
\end{array}\right), \quad g_{j}=\left\{\begin{array}{l}
1,-n+1 \leqslant j \leqslant n, \\
0, j<-n+1, \quad j>n .
\end{array}\right.
$$

Гамильтониан бильярда $V_{\mathrm{box}}$ мы включили в оператор возмущения, так как это ядерньй оператор.

При $|j| \gg n$ решение $u_{q}$ задачи рассеяния для оператора $H_{q}$ есть вектор-столбец со строками

$$
\left(u_{q}(\lambda, \pm, j)\right)_{1}=0, \quad\left(u_{q}(\lambda, \pm, j)\right)_{2}=e^{ \pm i k(\lambda) j}+w_{q}(\lambda, \pm, j),
$$

где функция $w_{q}$ Удовлетворяет условиям излучения (20). Коэффициенты прохождения и отражения в (20) (далее мы будем помечать их индексами $q$ ) - это те величины, которые мы исследуем.

При $q=\infty$ гамильтониан $H_{\infty}$ есть прямая сумма гамильтонианов $H_{0}$ и $V_{\mathrm{box}}$, а собственные значения оператора $V_{\mathrm{box}}$ есть погруженные в непрерывньй спектр собственные значения оператора $H_{\infty}$. При $\epsilon_{q}>0$ эти собственные значения преврашаются в "резонансы Фано", и нам требуется проанализировать поведение амплитуды рассеяния (т.е. коэффишиентов прохождения и отражения) вблизи резонанса при $q \rightarrow \infty$. Для этого мы воспользуемся предложенной в работах [3], [4] схемой. Условия, которые достаточны для применимости этой схемы, можно разделить на три группы.

1. Условия технического характера (полная непрерывность, ядерность рассматриваемых операторов и т.д.). В нашем случае невозмушенный гамильтониан ограничен, а возмушение конечномерно, так что справедливость всех этих условий тривиальна.

2. В работах [3], [4] предполагалось, что возмушение сдвигает погруженные собственные значения в комплексную плоскость. В нашем случае это условие можно проверить по "золотому правилу Ферми". Все величины, которые нужно подставить в известную формулу, выписаны выше. Рассматриваемая нами модель удовлетворяет условиям, при которых в работе [10] доказана формула (9.35), что гарантирует корректность применимости этого правила. 
3. Для того чтобы полюс с малой мнимой частью действительно порождал резонанс амплитуды рассеяния, в работах [3], [4] налагалось дополнительное условие: требовалось, чтобы сушествовали такие отображения $l: \mathcal{H} \rightarrow \mathcal{H}$ и $p: \Omega \rightarrow \Omega$ пространства состояний $\mathcal{H}$ рассматриваемой системы и параметризуюшего амплитуду рассеяния пространства $\Omega$, соответственно, что выполнены условия

$$
\begin{aligned}
& \forall f: \quad l(\alpha f)=\bar{\alpha} l(f), l\left(\left(\lambda-H_{0}\right)^{-1} V_{q} f\right)=\left(\bar{\lambda}-H_{0}\right)^{-1} V_{q} l(f), \\
& \forall \lambda \in \mathbb{C}, \quad \omega \in \Omega: \quad\left|\left\langle V_{q} l^{-1}(f), U^{-1}(\lambda, p(\omega))\right\rangle\right|=\left|U\left(V_{q} f\right)(\lambda, \omega)\right|,
\end{aligned}
$$

где $U^{-1}(\lambda, p(\omega))$ - интегральное ядро преобразования, обратного к преобразованию $U$.

Непосредственным выгислением можно проверить, что в случае параллельного соединения бильярда с проводником условию (41б) удовлетворяют отображения

$$
l(f)(j)=\overline{f(j)}, \quad p(+)=-, \quad p(-)=+,
$$

а в случае последовательного соединения бильярда с проводником условию (41б) удовлетворяют отображения

$$
l(f)(j)=\overline{f(-j)}, \quad p(+)=+, \quad p(-)=-.
$$

В последнем случае существенно используется симметрия системы относительно инверсии.

Рассмотрим сначала случай параллельного соединения бильярда и проводника. Пусть $\lambda_{m}$ - одно из не равных нулю собственных значений (36). Ясно, что в случае параллельного соединения бильярда и проводника при $q=\infty$ (бильярд не присоединен к проводнику) выполнены равенства $r_{\infty}(+, \lambda)=r_{\infty}(-, \lambda)=0$, поэтому из формул $(25)$ и (26) работы [3] следует

ТЕОрема 3. 1. При достаточно мальх $\epsilon_{q}$ в окрестности точки $\lambda_{m}$ существует полюс $\lambda_{m, q}$ матриць рассеяния $S\left(H_{q}, H_{0}\right)(\lambda) u$

$$
\operatorname{Im} \lambda_{m, q}<0, \quad \lambda_{m, q} \rightarrow \lambda_{m}, \quad q \rightarrow \infty
$$

2. Если $\nu_{q}-$ такая числовая последовательность, что

$$
\left|\nu_{q}-\operatorname{Re} \lambda_{m, q}\right|=O\left(\left|\operatorname{Im} \lambda_{m, q}\right|^{1+\delta}\right), \quad \delta>0,
$$

то коэффициенты отражения $r_{q}( \pm, \lambda)$ решения задачи рассеяния для оператора $H_{q}$ удовлетворяют соотношению

$$
\left|r_{q}\left( \pm, \nu_{q}\right)\right| \rightarrow 1, \quad q \rightarrow \infty
$$

Теперь рассмотрим случай последовательного соединения бильярда с проводником. Ясно, что в случае последовательного соединения бильярда с проводником при $q=\infty$ (проводник разомкнут и бильярд не присоединен к проводнику) выполнены равенства $t_{\infty}(+, \lambda)=t_{\infty}(-, \lambda)=0$, поэтому из формул $(25)$ и $(26)$ работы [3] следует 
Теорема 4. 1. При достаточно мальх $\epsilon_{q}$ в окрестности точки $\lambda_{m}$ существует полюс $\lambda_{m, q}$ матрииъ рассеяния $S\left(H_{q}, H_{0}\right)(\lambda) u$

$$
\operatorname{Im} \lambda_{m, q}<0, \quad \lambda_{m, q} \rightarrow \lambda_{m}, \quad q \rightarrow \infty .
$$

2. Если $\nu_{q}-$ такая числовая последовательность, что

$$
\left|\nu_{q}-\operatorname{Re} \lambda_{m, q}\right|=O\left(\left|\operatorname{Im} \lambda_{m, q}\right|^{1+\delta}\right), \quad \delta>0,
$$

то коэффичиенты прохождения $t_{q}( \pm, \lambda)$ решения задачи рассеяния для оператора $H_{q}$ удовлетворяют соотношению

$$
\left|t_{q}\left( \pm, \nu_{q}\right)\right| \rightarrow 1, \quad q \rightarrow \infty
$$

Теоремы 3 и 4 являются математической формулировкой сформулированных во введении утверждений.

На интуитивном уровне понятно, что в теоремах 3 и 4 нетривиально только утверждение о том, что обязательно должен быть скачок амплитуды рассеяния. Ясно, что величина скачка не может быть другой.

С математической точки зрения обсуждаемая нами проблема связана с теорией возмущений погруженных в непрерывный спектр собственных значений. В физике эта задача обычно решается методом введения эффективного гамильтониана [2], [11], [12]. Метод эффективного гамильтониана позволяет получить детальную информацию о поведении возмушенной связанной системы, но он требует дополнительных предположений. Предлагаемая нами модель является вариантом хорошо известной схемы (см., например, [10]) и имеет более скромные цели: она предназначена для анализа резонансов рассеяния в фиксированном радиационном канале. Заметим, что мы изучаем только полюсы первого порядка, более сложные особенности матрицы рассеяния нами не рассматриваются, и мы опираемся только на свойства симметрии системы.

Благодарности. Работа вьполнена при поддержке гранта РФФИ № 02-01-00271.

\section{Список литературы}

[1] B. Ricco, M. Ya. Azbel. Phys. Rev. B. 1984. V. 29. P. 1970.

[2] A. F. Sadreev, I. Rotter. J. Phys. A. 2003. V. 36. P. 11413; quant-ph/0304147.

[3] А. А. Арсеньев. ТМФ. 2003. Т. 136. № 3. С. 507.

[4] А.А. Арсеньев. ТМФ. 2003. Т. 134. № 3. С. 341.

[5] M. E. Torio, K. Hallberg, C. Proetto. Charge and spin conductance through a side-coupled quantum dot. cond-math/0404146.

[6] A. A. Arseniev. arseniev.zip на http://afrodita.phys.msu.su/persons/arsenev/docs.

[7] И. Ц. Гохберг, М. Г. Крейн. Введение в теорию линейных несамосопряженных операторов. М.: Наука, 1965.

[8] Д. Р. Яфаев. Математическая теория рассеяния. С.-Пб.: Изд-во СПбГУ, 1994.

[9] T. Ando. Phys. Rev. B. 1991. V. 44. № 15. P. 8017.

[10] S. Agmon. Commun. Pure Appl. Math. 1984. V. 101. P. 1084.

[11] F.-M. Dittes. Phys. Rep. 2000. V. 339. P. 215.

[12] J. Okolowicz, M. Ptoszajczak, I. Rotter. Phys. Rep. 2003. V. 374. P. 271.

Поступила в редакцию 16.I.2004 г., после доработки 4.III.2004 г. 